\title{
Automatic DNA sequencing
}

\author{
Akiyoshi Wada describes a system that takes the grind out of DNA sequencing
}

DNA SEQUENCING analysis is of fundamental importance today in pure and applied molecular biology. But the current procedure for analysing DNA sequences is both tedious and time-consuming. In the case of the Maxam-Gilbert scheme, more than thirty steps of microchemical manipulation are involved and these can only be carried out manually by a skilled chemist.

However, even using manual procedures the world-wide rate of analysis may exceed $10^{6}$ bases per year soon, judging by the current rate of additions to the DNA database. But this seemingly high rate of input is far below what is needed to meet the expected demands of sequence analysis in the future. There are many genomes still to be sequenced and even a single human genome has $3 \times 10^{9}$ bases.

An automated DNA sequencer recently developed by Seiko Instruments and Electronics of Tokyo, Japan, should take some of the drudgery out of DNA sequencing and speed up data production. The instrument is a microchemical robot that can carry out the fragmentations of 16 DNA solutions - that is it can test for the four bases ( $A, G, C$ and $T$ ) in each of four different DNA solutions. It performs the fragmentation in four to five hours, following the Maxam-Gilbert protocol. In

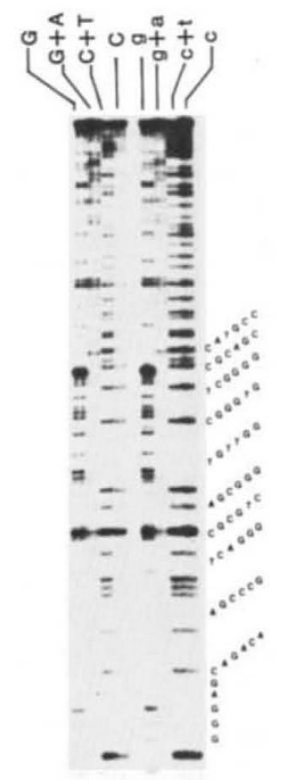

Comparison of sequencing patterns prepared manually (left) and prepared with the sequencer (right). addition, it can carry out two sets of operations sequentially so that 10 hours after the start button is pushed, 32 solutions are prepared for electrophoresis. The analysis may be started in the evening as the laboratory closes and then processed material can be collected the next morning.

The fully automatic chemical operations performed by this Seiko robotic instrument include the quantitative addition of reagents, mixing by vortexing, centrifugal separation, draining, vacuum drying, and the heating and cooling of solutions under treatment. The volume of solutions to be handled can be varied from $1 \mu \mathrm{l}$ to $1 \mathrm{ml}$. The time schedule and conditions of reaction steps in a series of operations are controlled by a microcomputer command which is input by the operator before the start of the sequencing process. Programmable functions extend this microchemical robot's range of performance to include any of a series of complex chemical reactions of microliter order ${ }^{1}$.

The instrument automates just one aspect of the DNA sequencing procedure and will earn a niche in the market by virtue of its labour-saving capacity rather than by any change in the speed or economy of sequencing. The time it takes to determine a sequence is only a little less than that need- ed to complete a sequence manually, the sequencer runs the same number of solutions as would a skilled technician and it does not save on reagents. But its designers believe that because it can run for 10 hours when the laboratory is effectively closed and because it frees the chemist to carry out other aspects of the sequencing procedure, it will be commercially viable. Tests have shown that the instrument can perform chemical reactions at the same level of efficiency and reliability as a skilled technician. What is more, the automatic sequencer requires no training and works steadily for 24 hours a day without complaining - something which should make it very attractive to the potential buyer!

The machine should be commercially available later this year, although details of the instrument's price and its exact date of launch have not yet been released.

Akiyoshi Wada is professor of biophysics in the Department of Physics, University of Tokyo and the chairman of the project 'DNA extraction, analysis and synthesis'. The DNA sequencer was developed as part of this project under the auspices of the Science and Technology Council of Japan.

1. Wada, A., Yamamoto, M. and Soeda, E. Rev. Sci. Instr. (in the press).

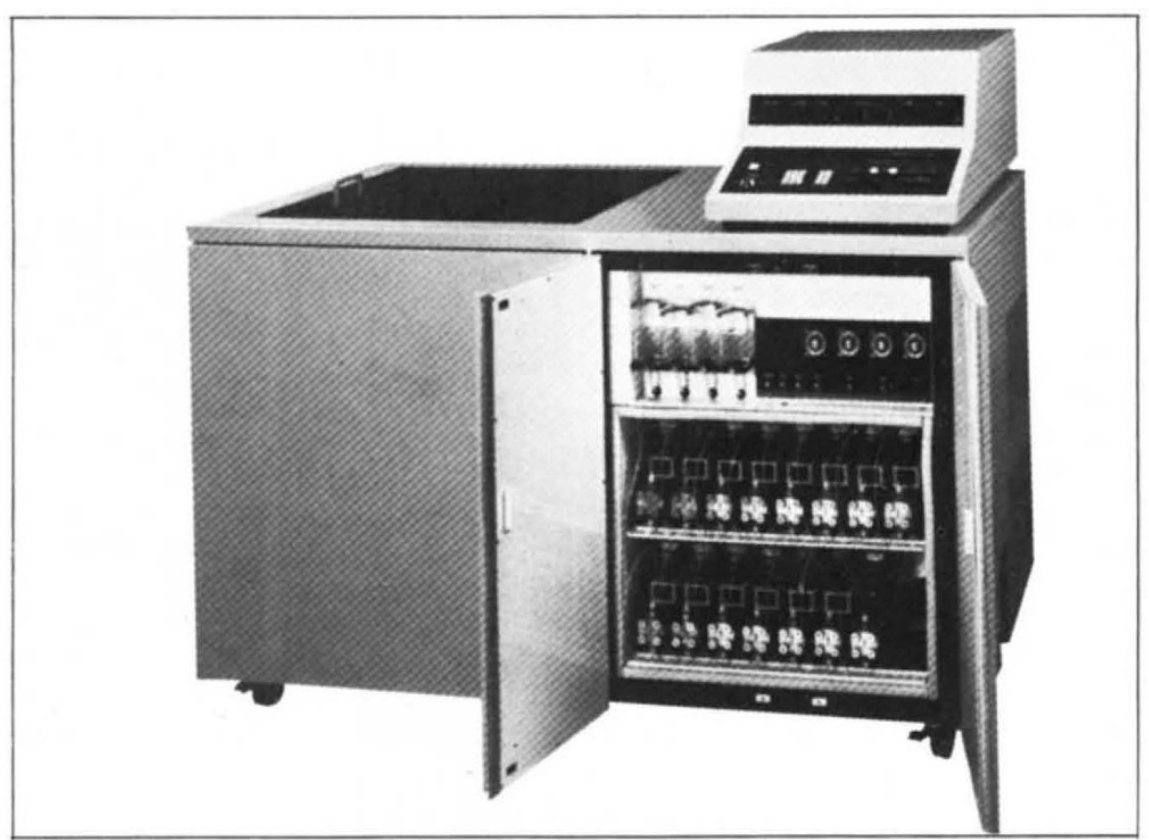

View of the microchemical robot. The computer system is at the top of the desk and the temperature control dial, stock solutions and pump systems, which are normally under cover, can be seen inside. 